\title{
Association between Body Fat and Diabetic Peripheral Neuropathy in Middle-Aged Adults with Type 2 Diabetes Mellitus: A Preliminary Report
}

\author{
Tae Jung Oh*, Jie-Eun Lee, Sung Hee Choi, Hak Chul Jang \\ Department of Internal Medicine, Seoul National University College of Medicine, Seoul; Department of Internal Medicine, Seoul National University Bundang \\ Hospital, Seongnam, Korea
}

\begin{abstract}
Background: Previous epidemiologic studies showed that obesity increased the risk of diabetic peripheral neuropathy (DPN). However, there is very limited data about the impact of body fat measured by body composition analysis in DPN.

Methods: Subjects with type 2 diabetes mellitus (T2DM) between 20 to 55 years old were enrolled. DPN was diagnosed using the Michigan Neuropathy Screening Instrument. Body composition was assessed by bio-impedance analysis, and the association between body composition and DPN was investigated.

Results: Among 65 subjects, 44.6\% were diagnosed with DPN. Subjects with DPN had higher body mass index and waist circumference than subjects without DPN. Body composition data showed that fat mass, fat percent, and visceral fat area were higher in subjects with DPN than in subjects without DPN. Furthermore, the presence of DPN was associated with waist circumference (odds ratio [OR], 1.151; 95\% confidence interval [Cl], 1.0551.256; $P=0.002)$, visceral fat area $(\mathrm{OR}, 1.026 ; 95 \% \mathrm{Cl}, 1.005-1.048 ; P=0.015)$, and insulin resistance $(\mathrm{OR}, 1.673$; $95 \% \mathrm{Cl}, 1.091-2.565 ; P=0.018$ ) after adjusting age, sex, diabetes duration, and smoking status.

Conclusion: Abdominal obesity was associated with DPN. Insulin resistance might mediate obesity and DPN in middle aged subjects with T2DM.
\end{abstract}

Key words: Diabetic neuropathy, Obesity, Visceral fat, Insulin resistance
Received February 25, 2019

Reviewed March 13, 2019

Accepted May 13, 2019

*Corresponding author

Tae Jung Oh

(1)

https://orcid.org/0000-0002-5078-6123

Department of Internal Medicine, Seoul National University College of Medicine, Seoul; Department of Internal Medicine, Seoul National University Bundang Hospital, 82 Gumi-ro 173beon-gil, Bundang-gu, Seongnam 13620, Korea Tel: +82-31-787-7078

Fax: +82-31-787-4052

E-mail: ohtjmd@gmail.com

\section{INTRODUCTION}

Diabetic peripheral neuropathy (DPN) is a prevalent and progressive microvascular complication of diabetes. ${ }^{1}$ Early detection and prevention are essential in reducing DPN-related morbidity and mortality. To do this, risk factors of DPN must be identified. According to previous epidemiologic studies ${ }^{2,3}$ and intervention studies ${ }^{4}$, age, diabetes duration, smoking status, and components of metabolic syndrome including obesity are well-known risk factors for DPN.

There are five components of metabolic syndrome: central obesity, high blood pressure, low high-density lipoprotein (HDL) cholesterol, high triglycerides, and hyperglycemia. ${ }^{5}$ Patients with diabe- tes already have hyperglycemia, but other modifiable risk factors such as hypertension, dyslipidemia, or central obesity can be addressed. Central obesity with insulin resistance is a key pathophysiologic factor of metabolic syndrome, so assessment of the relationship between obesity and insulin resistance should be investigated. The Anglo-Danish-Dutch study of Intensive Treatment of Diabetes in Primary Care (ADDITION) study ${ }^{6}$ and Cooperative Health Research in the Region of Augsburg (KORA) study ${ }^{7}$ consistently demonstrated that general and central obesity increased the risk of DPN. However, these well-designed prospective observational studies did not assess body composition other than measurement of waist circumference (WC). Furthermore, the ADDITION and

Copyright (C) 2019 Korean Society for the Study of Obesity

(a) This is an Open Access article distributed under the terms of the Creative Commons Attribution Non-Commercial License (http://creativecommons.org/licenses/by-nc/4.0/) which permits unrestricted non-commercial use, distribution, and reproduction in any medium, provided the original work is properly cited. 
KORA studies enrolled patients that were older than 60 and 65 years, respectively. Body composition is influenced by menopause (or andropause for men) ${ }^{8,9}$ and age-related sarcopenia. ${ }^{10}$ Therefore, we enrolled young to middle adults to minimize the effect of aging on body composition. This study investigated the association between body composition, components of metabolic syndrome, and DPN in middle-aged adults with type 2 diabetes mellitus (T2DM). We hypothesized that body fat was highly associated with the presence of DPN.

\section{METHODS}

\section{Subjects}

Subjects diagnosed with T2DM were recruited. This is a subgroup analysis of an ongoing prospective observational study. The study plans to enroll 100 subjects annually, and there will be $500 \mathrm{fi}-$ nal participants with T2DM. The study has been designed to investigate reliable tools and discover biomarkers for DPN. The current analysis was performed using subgroup data for the initial 2-year enrolled participants. Inclusion criteria of current analysis included age from 20 to 55 years and no change of antidiabetic medications in the previous 3 months. Exclusion criteria were other causes of neuropathy such as heavy alcohol consumption, renal dysfunction (estimated glomerular filtration rate less than $50 \mathrm{~mL} / \mathrm{min} / 1.73 \mathrm{~m}^{2}$ ), any history of cancer, and exposure to neurotoxic agents. The study was approved by the Institutional Review Board of Seoul National University Bundang Hospital (IRB No. B-1903/526-101). All participants provided written informed consent.

\section{Assessment of DPN}

DPN was diagnosed using the Michigan Neuropathy Screening Instrument $(\mathrm{MNSI})^{11}$, which was validated in Korean patients with diabetes. ${ }^{12}$ The MNSI is composed of two components, a 15-item self-administered questionnaire and a comprehensive physical examination. DPN was diagnosed when the MNSI-questionnaire (MNSI-Q) was $\geq 7$ or MNSI physical examination was $\geq 4$.

\section{Anthropometric and body composition measurements}

Body weight, height, WC, and blood pressure were measured by a single trained research nurse. All anthropometric examination was performed in light clothing with bare feet. Body mass index (BMI) was calculated as body weight $(\mathrm{kg}) /$ height $\left(\mathrm{m}^{2}\right)$. WC was obtained at the middle of the lower rib margin and iliac crest. Systolic and diastolic blood pressure (SBP/DBP) were measured after a 10-minute rest using an electronic blood pressure meter (UA1020 device; A\&D Co., Tokyo, Japan). Total body fat mass, muscle mass, and visceral fat area were measured by bio-impedance analysis (InBody770; InBody, Seoul, Korea).

\section{Biochemical analysis}

Participants visited the Endocrine Clinic after fasting overnight. Blood glucose levels were measured by the hexokinase method, and glycosylated hemoglobin (HbAlc) levels were obtained by high-performance liquid chromatography (Bio-Rad, Hercules, CA, USA). Fasting insulin levels were measured by immunoradiometric assay (DIAsource, Nivelles, Belgium). Homogeneous enzymatic assays and glycerol-3-phosphate oxidase peroxide methods were used to measure HDL cholesterol, low-density lipoprotein cholesterol, and triglyceride. Liver and renal function tests were performed using the protocol of the central laboratory of Seoul $\mathrm{Na}$ tional University Bundang Hospital. Homeostatic model assessment for insulin resistance (HOMA-IR) was calculated as an indicator of insulin resistance using the following formula: plasma insulin $(\mu \mathrm{IU} / \mathrm{mL}) \times$ plasma glucose $(\mathrm{mg} / \mathrm{dL}) / 405 .{ }^{13}$ Insulin resistant status was defined as when HOMA-IR level was higher than 3.0, according to a previous study. ${ }^{14}$

\section{Statistical analysis}

Data are shown as mean \pm standard deviation or number with percentage. Means were compared between subjects with and without DPN using Student t-test or the Mann-Whitney U-test. Differences in sex, history of smoking, alcohol, hypertension, dyslipidemia, and antidiabetic medications were tested by chi-square test. Spearman's correlation analysis was performed to evaluate the association between neuropathy examination results, metabolic syndrome components, and body composition. Univariate and multivariate logistic regression models were used to estimate the association of clinical parameters and presence of DPN using odds ratios (ORs) with 95\% confidential intervals (CIs). Statistical analysis was performed using IBM SPSS version 22.0 (IBM Corp., Armonk, NY, USA). 


\section{RESULTS}

In this study, data from 65 subjects with T2DM was analyzed. Among 65 subjects, $44.6 \%$ were diagnosed with DPN. There was no difference in age or sex between subjects without DPN and those with DPN. BMI was higher in subjects with DPN than in subjects without DPN $\left(26.5 \pm 4.3 \mathrm{~kg} / \mathrm{m}^{2}\right.$ vs. $\left.24.1 \pm 3.2 \mathrm{~kg} / \mathrm{m}^{2}, P=0.011\right)$.

Table 1. Clinical and biochemical characteristics of patients stratified by presence of DPN

\begin{tabular}{|c|c|c|c|}
\hline Variable & $\operatorname{DPN}(-)(n=36)$ & DPN $(+)(n=29)$ & $P$ \\
\hline Male sex & $25(69.4)$ & $18(62.1)$ & 0.603 \\
\hline Age (yr) & $47.3 \pm 5.6$ & $47.9 \pm 8.0$ & 0.730 \\
\hline Height (cm) & $168.2 \pm 9.2$ & $164.2 \pm 9.4$ & 0.092 \\
\hline Body weight (kg) & $68.7 \pm 13.5$ & $71.4 \pm 14.7$ & 0.438 \\
\hline $\mathrm{BMI}\left(\mathrm{kg} / \mathrm{m}^{2}\right)$ & $24.1 \pm 3.2$ & $26.5 \pm 4.3$ & 0.011 \\
\hline $\mathrm{SBP}(\mathrm{mmHg})$ & $128.8 \pm 10.6$ & $134.8 \pm 14.8$ & 0.063 \\
\hline $\mathrm{DBP}(\mathrm{mmHg})$ & $77.1 \pm 7.7$ & $79.3 \pm 9.7$ & 0.304 \\
\hline Diabetes duration (yr) & $7.3 \pm 5.0$ & $10.0 \pm 8.3$ & 0.144 \\
\hline Glucose (mg/dL) & $139.3 \pm 29.6$ & $156.0 \pm 57.9$ & 0.165 \\
\hline $\mathrm{HbA1c}(\%)$ & $7.2 \pm 1.2$ & $7.8 \pm 1.8$ & 0.111 \\
\hline Cholesterol (mg/dL) & $169.0 \pm 42.1$ & $160.8 \pm 52.3$ & 0.486 \\
\hline Triglyceride (mg/dL) & $160.6 \pm 156.9$ & $208.6 \pm 248.0$ & 0.417 \\
\hline HDL cholesterol (mg/dL) & $50.4 \pm 12.2$ & $47.0 \pm 16.7$ & 0.347 \\
\hline LDL cholesterol (mg/dL) & $95.3 \pm 25.8$ & $88.5 \pm 32.9$ & 0.352 \\
\hline BUN (mg/dL) & $15.7 \pm 3.4$ & $15.8 \pm 4.8$ & 0.945 \\
\hline Creatinine (mg/dL) & $0.7 \pm 0.2$ & $0.8 \pm 0.2$ & 0.288 \\
\hline $\mathrm{eGFR}\left(\mathrm{mL} / \mathrm{min} / 1.73 \mathrm{~m}^{2}\right)$ & $108.7 \pm 23.0$ & $100.5 \pm 24.2$ & 0.168 \\
\hline AST (IU/L) & $26.9 \pm 15.3$ & $35.1 \pm 19.2$ & 0.004 \\
\hline ALT (IU/L) & $27.8 \pm 19.5$ & $38.4 \pm 22.3$ & 0.045 \\
\hline Insulin & $6.5 \pm 3.0$ & $8.9 \pm 5.0$ & 0.093 \\
\hline HOMA-IR & $2.3 \pm 1.2$ & $3.4 \pm 2.1$ & 0.024 \\
\hline MNSI-0 (score) & $2.0 \pm 1.7$ & $3.6 \pm 2.8$ & 0.021 \\
\hline MNSI-PE (score) & $1.3 \pm 0.7$ & $3.4 \pm 1.0$ & $<0.001$ \\
\hline Smoking status & & & 0.074 \\
\hline Never smoker & $10(27.8)$ & $16(55.2)$ & \\
\hline Ex-smoker & $12(33.3)$ & $5(17.2)$ & \\
\hline Current smoker & $14(38.9)$ & $8(29.6)$ & \\
\hline Alcohol & $23(63.9)$ & $15(51.7)$ & 0.448 \\
\hline Hypertension & $22(61.1)$ & $21(72.4)$ & 0.432 \\
\hline Dyslipidemia & $21(58.3)$ & $19(65.5)$ & 0.614 \\
\hline Oral antidiabetic medication & 32 (88.9) & 27 (93.1) & 0.684 \\
\hline Insulin therapy & $5(13.9)$ & $8(27.6)$ & 0.218 \\
\hline
\end{tabular}

Values are presented as number (\%) or mean \pm standard deviation.

DPN, diabetic peripheral neuropathy; BMl, body mass index; SBP, systolic blood pressure; DBP, diastolic blood pressure; HbA1c, glycosylated hemoglobin; HDL, high-density lipoprotein; LDL, low-density lipoprotein; BUN, blood urea nitrogen; eGFR, estimated glomerular filtration rate; AST, aspartate aminotransferase; ALT, alanine aminotransferase; HOMA-IR, homeostatic model assessment for insulin resistance; MNSI-0, Michigan Neuropathy Screening Instrument-questionnaire; MNSI-PE, MNSI-physical examination.
SBP tended to be higher in the DPN (+) group, but lipid profiles were comparable between groups. HOMA-IR was significantly increased in the DPN (+) group compared to the DPN (-) group. There was a numerically longer duration of diabetes in the DPN $(+)$ group than the DPN (-) group, and subjects with DPN were more likely to be treated with insulin than subjects without DPN (Table 1).

Table 2 shows body composition data. WC, fat mass, fat percent, and visceral fat area were significantly increased in the DPN $(+)$ group than the DPN (-) group. However, lean body mass was not different between groups.

MNSI-physical examination (MNSI-PE) scores were positively correlated with $\mathrm{HbA1c}$ (rho $=0.261, P=0.035)$, SBP $(\mathrm{rho}=0.300$, $P=0.017)$, and HOMA-IR $(\mathrm{rho}=0.310, P=0.017)$. However, these

Table 2. Body composition analysis data of patients by presence of DPN

\begin{tabular}{lccc}
\hline Variable & DPN $(-)(\mathrm{n}=36)$ & $\mathrm{DPN}(+)(\mathrm{n}=29)$ & $P$ \\
\hline WC $(\mathrm{cm})$ & $82.9 \pm 9.6$ & $89.0 \pm 7.9$ & 0.008 \\
Fat mass $(\mathrm{kg})$ & $17.2 \pm 6.4$ & $21.9 \pm 8.8$ & 0.016 \\
Fat percent $(\%)$ & $25.0 \pm 7.6$ & $30.0 \pm 7.7$ & 0.010 \\
Lean body mass $(\mathrm{kg})$ & $48.5 \pm 10.2$ & $47.0 \pm 9.0$ & 0.539 \\
Visceral fat area $\left(\mathrm{cm}^{2}\right)$ & $78.4 \pm 31.6$ & $102.0 \pm 43.3$ & 0.014
\end{tabular}

Values are presented as mean \pm standard deviation.

DPN, diabetic peripheral neuropathy; WC, waist circumference.

Table 3. Correlation coefficients of Spearman analysis between MNSI, metabolic syndrome components, and body composition data

\begin{tabular}{lcccccc}
\hline \multirow{2}{*}{ Variable } & \multicolumn{2}{c}{ MNSI-0 } & & \multicolumn{2}{c}{ MNSI-PE } \\
\cline { 2 - 3 } \cline { 6 - 7 } & rho & $P$ & & rho & $P$ \\
\hline HbA1c $(\%)$ & 0.045 & 0.720 & & 0.261 & 0.035 \\
Diabetes duration $(\mathrm{yr})$ & 0.083 & 0.516 & & 0.087 & 0.493 \\
Triglyceride $(\mathrm{mg} / \mathrm{dL})$ & 0.069 & 0.585 & & 0.264 & 0.034 \\
HDL cholesterol $(\mathrm{mg} / \mathrm{dL})$ & -0.067 & 0.595 & & -0.208 & 0.097 \\
SBP $(\mathrm{mmHg})$ & -0.063 & 0.623 & & 0.300 & 0.017 \\
DBP $(\mathrm{mmHg})$ & -0.177 & 0.164 & & 0.253 & 0.045 \\
HOMA-IR & -0.005 & 0.972 & & 0.310 & 0.017 \\
WC $(\mathrm{cm})$ & 0.085 & 0.505 & & 0.364 & 0.003 \\
BMI $\left(\mathrm{kg} / \mathrm{m}^{2}\right)$ & -0.159 & 0.207 & & 0.348 & 0.005 \\
Fat mass $(\mathrm{kg})$ & -0.032 & 0.799 & & 0.357 & 0.004 \\
Fat percent $(\%)$ & -0.021 & 0.870 & & 0.332 & 0.007 \\
Lean body mass $(\mathrm{kg})$ & -0.165 & 0.190 & & -0.067 & 0.597 \\
Visceral fat area $\left(\mathrm{cm}^{2}\right)$ & -0.006 & 0.190 & & 0.330 & 0.007 \\
\hline
\end{tabular}

MNSI, Michigan Neuropathy Screening Instrument; MNSI-Q, MNSI-questionnaire; MNSI-PE, MNSI-physical examination; HbA1c, glycosylated hemoglobin; HDL, highdensity lipoprotein; SBP, systolic blood pressure; DBP, diastolic blood pressure; HOMAIR, homeostatic model assessment for insulin resistance; WC, waist circumference; $\mathrm{BMl}$, body mass index. 
Table 4. Odds ratios and 95\% confidential intervals of presence of DPN according to metabolic and anthropometric components

\begin{tabular}{lcccc}
\hline Variable & Unadjusted model & $P$ & Adjusted model & $P$ \\
\hline Glucose $(\mathrm{mg} / \mathrm{dL})$ & $1.009(0.997-1.021)$ & 0.147 & $1.009(0.996-1.023)$ & 0.160 \\
HbA1c $(\%)$ & $1.336(0.940-1.899)$ & 0.107 & $1.394(0.932-2.084)$ & 0.106 \\
Triglyceride $(\mathrm{mg} / \mathrm{dL})$ & $1.001(0.999-1.004)$ & 0.360 & $1.002(0.999-1.005)$ & 0.104 \\
$\mathrm{HDL}$ cholesterol $(\mathrm{mg} / \mathrm{dL})$ & $0.983(0.947-1.019)$ & 0.347 & $0.978(0.938-1.019)$ & 0.281 \\
$\mathrm{SBP}(\mathrm{mmH} \mathrm{Hg})$ & $1.040(0.997-1.085)$ & 0.069 & $1.056(1.004-1.111)$ & 0.034 \\
$\mathrm{DBP}(\mathrm{mmHg})$ & $1.032(0.973-1.095)$ & 0.300 & $1.067(0.985-1.155)$ & 0.111 \\
$\mathrm{HOMA}-\mathrm{IR}$ & $1.561(1.079-2.258)$ & 0.018 & $1.673(1.091-2.565)$ & 0.018 \\
$\mathrm{WC}(\mathrm{cm})$ & $1.086(1.018-1.158)$ & 0.013 & $1.151(1.055-1.256)$ & 0.002 \\
BMI $\left(\mathrm{kg} / \mathrm{m}^{2}\right)$ & $1.210(1.033-1.417)$ & 0.018 & $1.266(1.045-1.534)$ & 0.016 \\
Fat mass $(\mathrm{kg})$ & $1.095(1.012-1.186)$ & 0.025 & $1.131(1.021-1.252)$ & 0.018 \\
Fat percent $(\%)$ & $1.091(1.018-1.170)$ & 0.014 & $1.147(1.030-1.277)$ & 0.013 \\
Lean body mass $(\mathrm{kg})$ & $0.984(0.934-1.036)$ & 0.533 & $0.988(0.896-1.090)$ & 0.811 \\
Visceral fat area $\left(\mathrm{cm}^{2}\right)$ & $1.018(1.003-1.035)$ & 0.022 & $1.026(1.005-1.048)$ & 0.015
\end{tabular}

Values are presented as odds ratio ( $95 \%$ confidential interval). Adjusted model was adjusted for age, sex, diabetes duration, and smoking status.

DPN, diabetic peripheral neuropathy; HbA1c, glycosylated hemoglobin; HDL, high-density lipoprotein; SBP, systolic blood pressure; DBP, diastolic blood pressure; HOMA-IR, homeostatic model assessment for insulin resistance; WC, waist circumference; BMI, body mass index.

parameters did not show significant association with MNSI-Q. Among body composition parameters, total obesity and abdominal obesity were positively correlated with MNSI-PE (Table 3).

Univariate regression analysis revealed that HOMA-IR (OR, 1.561 ; 95\% CI, 1.079-2.258; $P=0.018)$, WC (OR, 1.086; 95\% CI, 1.018-1.158; $P=0.013)$, BMI (OR, 1.210; 95\% CI, 1.033-1.417; $P=0.018)$, and visceral fat area (OR, 1.018; 95\% CI, 1.003-1.035; $P=0.022$ ) were associated with the presence of DPN. Significance was maintained after adjusting for age, sex, diabetes duration, and smoking status (Table 4).

When subjects were categorized according to their BMI and insulin resistance status, lean subjects with lower HOMA-IR were less likely to have DPN (18.5\%). In contrast, the majority of subjects who were obese and had higher insulin resistance also had DPN (66.7\%) (Fig. 1).

\section{DISCUSSION}

In this observational study that included young to middle-aged adults with T2DM, $44.6 \%$ of subjects were diagnosed with DPN according to MNSI. Subjects with DPN showed higher insulin resistance and a greater degree of general and abdominal obesity.

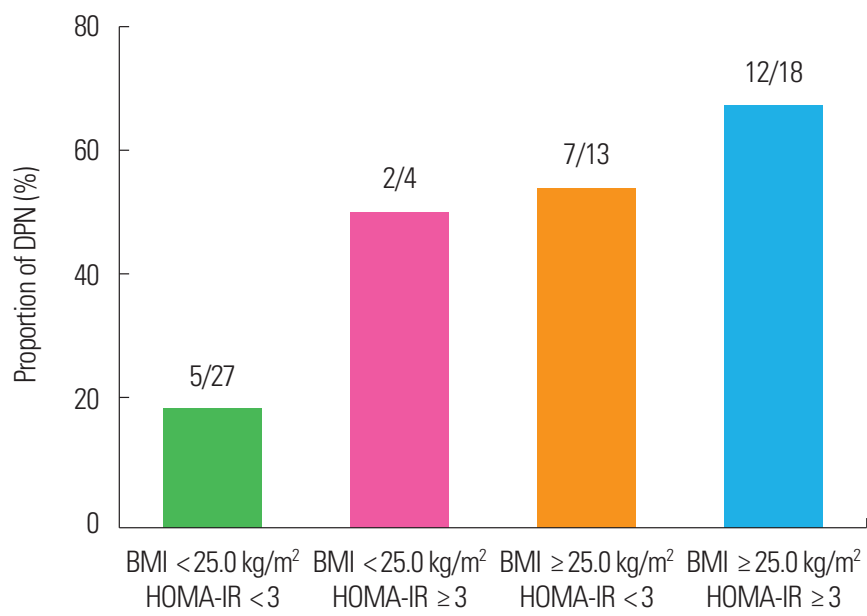

Figure 1. Proportion of diabetic peripheral neuropathy (DPN) according to body mass index (BMI) and homeostatic model assessment for insulin resistance (HOMA-IR). Values indicate number of subjects with DPN/total number of subgroups.

Furthermore, the physical examination score of subjects with DPN was significantly associated with HOMA-IR, BMI, WC, and visceral fat area.

Among the five components of metabolic syndrome, central obesity was the most important risk factor for DPN in subjects with diabetes. In our study, the parameters of central obesity, WC, and visceral fat area were highly correlated with HOMA-IR (rho = 0.510 for WC, $P<0.001$; rho $=0.366$ for visceral fat area, $P=0.008$ ). In addition, the statistically significant association between DPN and visceral fat area diminished after adjusting for HOMA-IR (data not shown). Therefore, insulin resistance might be a key regulator between obesity and DPN. Insulin resistant status represents low grade inflammation ${ }^{15}$, which influences endothelial dysfunction ${ }^{16}$ and microvascular complications. ${ }^{17,18}$ The KORA study proved that biomarkers of subclinical inflammation were associated with DPN progression. ${ }^{19}$ Our study provides evidence of the pathophysiologic role of abdominal obesity and insulin resistance in DPN. However, hyperglycemic-euglycemic clamp studies or oral glucose tolerance tests at multiple time points are needed to measure insulin resistance more accurately.

Previous intervention studies showed that body weight loss decreased the incidence of DPN. ${ }^{4,20}$ Although secondary effects due to improved hyperglycemia after body weight loss cannot be ruled out, body weight management is an important intervention strategy for DPN. Studies have already shown the beneficial effect of 
bariatric/metabolic surgery on cardiac and sudomotor dysfunction. ${ }^{21}$ However, we do not know whether other treatment modalities related to body weight gain, such as insulin therapy ${ }^{22}$, might contribute to DPN progression. In this study, a numerically higher proportion of subjects with DPN were treated with insulin than subjects without DPN. Further study is necessary to investigate the impact of insulin-induced weight gain on DPN progression.

Correlation analysis showed significant correlations only between obesity parameters and MNSI-PE. In contrast, there was no association between obesity parameters and MNSI-Q. Therefore, the only objective signs of DPN were related to metabolic parameters. Further investigation is needed to demonstrate the association between DPN and subjective symptoms. In line with our findings, physical examination might be more useful in clinical practice than questionnaire-based evaluation in subjects with obesity.

Previously, Won et al. ${ }^{23}$ reported that the prevalence of DPN was $33.5 \%$ in Korean subjects with T2DM. The previous study enrolled subjects from both secondary and tertiary hospitals, while we enrolled subjects from a single tertiary hospital that patients were referred to by primary care physicians. Furthermore, DPN criteria were not the same between previous and current studies. The previous study diagnosed DPN when both the MNSI-Q $(\geq 3)$ and 10-g monofilament test were abnormal, while we diagnosed DPN using the MNSI-Q $(\geq 7)$ and/or physical examination. The $10-\mathrm{g}$ monofilament test is recommended to detect loss of protective sensation ${ }^{24}$, rather than to diagnose DPN, so we did not adopt the 10-g monofilament test to diagnose DPN. In summary, differences in the study population and diagnostic criteria are likely related to the different prevalence of DPN in different studies. Further multicenter prospective observational studies are necessary to confirm our findings.

This study has several limitations. First, a relatively small sample was analyzed. Accordingly, no significant association was identified between DPN and its traditional risk factors, such as age and diabetes duration. The current analysis was performed in a subgroup sample from a whole cohort population, and further confirmative analysis is mandatory to confirm the study findings. Second, electrophysiologic study was not used to confirm DPN. However, some proportion of DPN cannot be diagnosed by nerve conduction studies (NCSs) because small fiber neuropathy is often not detected by NCSs. MNSI has been validated for the diagnosis of DPN and is widely used in many epidemiologic studies. Third, other symptom scores were not analyzed. Fourth, middle aged people were enrolled. Therefore, the association between DPN and body composition should be tested in elderly patients. The strengths of our study include assessment of abdominal obesity using not only anthropometric measurement but also bio-impedance analysis, although accuracy of bio-impedance analysis is limited with respect to measuring visceral fat area. ${ }^{25}$

In conclusion, total obesity and abdominal obesity were associated with DPN, and insulin resistance might mediate obesity and DPN. The current study provided important insight into DPN as a clinical parameter in obesity intervention studies.

\section{CONFLICTS OF INTEREST}

The authors declare no conflict of interest.

\section{REFERENCES}

1. Pop-Busui R, Boulton AJ, Feldman EL, Bril V, Freeman R, Malik RA, et al. Diabetic neuropathy: a position statement by the American Diabetes Association. Diabetes Care 2017;40: 136-54.

2. Tesfaye S, Chaturvedi N, Eaton SE, Ward JD, Manes C, Ionescu-Tirgoviste $\mathrm{C}$, et al. Vascular risk factors and diabetic neuropathy. N Engl J Med 2005;352:341-50.

3. Andersen ST, Witte DR, Fleischer J, Andersen H, Lauritzen T, Jørgensen ME, et al. Risk factors for the presence and progression of cardiovascular autonomic neuropathy in type 2 diabetes: ADDITION-Denmark. Diabetes Care 2018;41:2586-94.

4. Look AHEAD Research Group. Effects of a long-term lifestyle modification programme on peripheral neuropathy in overweight or obese adults with type 2 diabetes: the Look AHEAD study. Diabetologia 2017;60:980-8.

5. Alberti KG, Zimmet P, Shaw J. Metabolic syndrome: a new world-wide definition. A Consensus Statement from the International Diabetes Federation. Diabet Med 2006;23:469-80.

6. Andersen ST, Witte DR, Dalsgaard EM, Andersen H, Nawroth P, Fleming T, et al. Risk factors for incident diabetic poly- 
neuropathy in a cohort with screen-detected type 2 diabetes followed for 13 years: ADDITION-Denmark. Diabetes Care 2018;41:1068-75.

7. Schlesinger S, Herder C, Kannenberg JM, Huth C, CarstensenKirberg M, Rathmann W, et al. General and abdominal obesity and incident distal sensorimotor polyneuropathy: insights into inflammatory biomarkers as potential mediators in the KORA F4/FF4 cohort. Diabetes Care 2019;42:240-7.

8. Svendsen OL, Hassager C, Christiansen C. Age- and menopause-associated variations in body composition and fat distribution in healthy women as measured by dual-energy X-ray absorptiometry. Metabolism 1995;44:369-73.

9. Matsumoto AM. Andropause: clinical implications of the decline in serum testosterone levels with aging in men. J Gerontol A Biol Sci Med Sci 2002;57:M76-99.

10. Lee Y, Shin H, Vassy JL, Kim JT, Cho SI, Kang SM, et al. Comparison of regional body composition and its relation with cardiometabolic risk between BMI-matched young and old subjects. Atherosclerosis 2012;224:258-65.

11. Feldman EL, Stevens MJ, Thomas PK, Brown MB, Canal N, Greene DA. A practical two-step quantitative clinical and electrophysiological assessment for the diagnosis and staging of diabetic neuropathy. Diabetes Care 1994;17:1281-9.

12. Won JC, Im YJ, Lee JH, Kim CH, Kwon HS, Cha BY, et al. Clinical phenotype of diabetic peripheral neuropathy and relation to symptom patterns: cluster and factor analysis in patients with type 2 diabetes in Korea. J Diabetes Res 2017;2017: 5751687.

13. Matthews DR, Hosker JP, Rudenski AS, Naylor BA, Treacher DF, Turner RC. Homeostasis model assessment: insulin resistance and beta-cell function from fasting plasma glucose and insulin concentrations in man. Diabetologia 1985;28:412-9.

14. Kernan WN, Viscoli CM, Furie KL, Young LH, Inzucchi SE, Gorman M, et al. Pioglitazone after ischemic stroke or transient ischemic attack. N Engl J Med 2016;374:1321-31.

15. Dandona P, Aljada A, Bandyopadhyay A. Inflammation: the link between insulin resistance, obesity and diabetes. Trends Immunol 2004;25:4-7.

16. Muniyappa R, Sowers JR. Role of insulin resistance in endothelial dysfunction. Rev Endocr Metab Disord 2013;14:5-12.
17. Schram MT, Chaturvedi N, Schalkwijk CG, Fuller JH, Stehouwer CD; EURODIAB Prospective Complications Study Group. Markers of inflammation are cross-sectionally associated with microvascular complications and cardiovascular disease in type 1 diabetes: the EURODIAB Prospective Complications Study. Diabetologia 2005;48:370-8.

18. Nguyen DV, Shaw LC, Grant MB. Inflammation in the pathogenesis of microvascular complications in diabetes. Front Endocrinol (Lausanne) 2012;3:170.

19. Herder C, Kannenberg JM, Huth C, Carstensen-Kirberg M, Rathmann W, Koenig W, et al. Proinflammatory cytokines predict the incidence and progression of distal sensorimotor polyneuropathy: KORA F4/FF4 study. Diabetes Care 2017; 40:569-76.

20. Diabetes Prevention Program Research Group. Long-term effects of lifestyle intervention or metformin on diabetes development and microvascular complications over 15-year followup: the Diabetes Prevention Program Outcomes Study. Lancet Diabetes Endocrinol 2015;3:866-75.

21. Casellini CM, Parson HK, Hodges K, Edwards JF, Lieb DC, Wohlgemuth SD, et al. Bariatric surgery restores cardiac and sudomotor autonomic $\mathrm{C}$-fiber dysfunction towards normal in obese subjects with type 2 diabetes. PLoS One 2016;11: e0154211.

22. Russell-Jones D, Khan R. Insulin-associated weight gain in diabetes: causes, effects and coping strategies. Diabetes Obes Metab 2007;9:799-812.

23. Won JC, Kwon HS, Kim CH, Lee JH, Park TS, Ko KS, et al. Prevalence and clinical characteristics of diabetic peripheral neuropathy in hospital patients with type 2 diabetes in Korea. Diabet Med 2012;29:e290-6.

24. Boulton AJ, Armstrong DG, Albert SF, Frykberg RG, Hellman $\mathrm{R}$, Kirkman MS, et al. Comprehensive foot examination and risk assessment: a report of the task force of the foot care interest group of the American Diabetes Association, with endorsement by the American Association of Clinical Endocrinologists. Diabetes Care 2008;31:1679-85.

25. Shuster A, Patlas M, Pinthus JH, Mourtzakis M. The clinical importance of visceral adiposity: a critical review of methods for visceral adipose tissue analysis. Br J Radiol 2012;85:1-10. 